\title{
Detection of Abnormal Shadows in Low-dose CT Images Using CNN
}

\author{
Hiromu Ikeda, Tohru Kamiya \\ Department of Mechanical and Control Engineering, Kyushu Institute of Technology, 1-1 Sensui-cho, Tobata-ku, \\ Kitakyushu-shi, Fukuoka, 804-8550, Japan \\ Takatoshi Aoki \\ University of Occupational and Environmental Health, 1-1, Iseigaoka, Yahatanishi, Kitakyusyu-shi 807-8555, Japan
}

\begin{abstract}
CT imaging is a very effective way to detect abnormal shadows in the lungs. Considering the physical burden on the patient, it should be taken at a low dose. However, there are currently few studies using low-dose CT as compared to normal-dose CT. Therefore, it is necessary to develop a system to support the diagnosis of low-dose CT. To detect lung nodules and GGO from a low-dose CT image, we propose image processing techniques based on CNN. Our approach consists of two main elements. First, candidate abnormal shadow areas are extracted from low-dose CT images of the chest by threshold processing and filtering. Second, we implement a CNN model to classify candidate region on images. To improve identification accuracy in $\mathrm{CNN}$, we devised a method to add spatial information to the input image. In this paper, the proposed method is applied to 13 abnormal shadow images and 100 normal tissue images, and true positive ate of $84.6 \%$, false negative rate of $15.4 \%$ were achieved respectively.
\end{abstract}

Keywords: Low-dose CT, Computer aided diagnosis, Dual-ring filter, Convolutional neural network, input image processing

\section{Introduction}

According to the world health organization (WHO), cancer is the second leading cause of death worldwide, and is responsible for an estimated 9.6 million people in 2018. Among them, lung cancer accounting 2.09 million cases, and is the large number of deaths cause. It is ranked the most common causes of cancer death [1]. Early detection is important factor to reduce mortality from cancer. If the cases are detected and treated in early stage, the mortality rate from cancer may decrease. When the cancer is detected in early stage, it has high survival rate, low morbidity, low treatment costs and high potential for effective treatment. Also, detecting the cancer in early time and avoiding delays in treatment may make a huge difference in the lives of cancer patients.
CT imaging is often used for early detection of the disease. However, there are some problems in CT imaging, such as an increase in the burden of the reader due to the huge number of images, and a difference in diagnostic results due to differences in the experience of physicians. To avoid this problem, there are some approaches in medical imaging fields. The CAD system digitizes the medical data, quantifies and analyzes the medical data using a computer. As a result, physician can use the results of the CAD as a "second opinion". In addition, the low-dose CT used in this paper, and is less burdensome on the patient than the one taken at a normal dose, however the image quality is poor.

In recent years, deep learning has attracted a lot of attention in the field of artificial intelligence. Especially in the field of image recognition fields, convolutional 
neural networks (CNNs) have become the most important technology to classified a target from an image. The various CNN architectures are also becoming increasingly important in the medical imaging field. In this paper, we describe the development of a CAD system for the detection of abnormal shadows from low-dose CT images. In this approach, candidate abnormal shadow areas are extracted from $\mathrm{CT}$ images and the regions are inputted into the CNN to classify normal or not.

\section{Method}

In this system, we extract candidate abnormal shadow areas from CT images based on CNN. The details are shown in following. Figure 1 shows an overview of the CAD system which is built in this paper. Figure 2 shows the flow of the initial candidate region extraction.

\subsection{Extraction of initial candidate area}

Lung field areas are extracted from CT images. In this paper, the areas were extracted by binarization, labeling, and thresholding techniques. Next, emphasis was applied to make it easier to detect abnormal shadows. The details are shown in follows.

\section{A. Segmentation of lung area}

To segment region of interest (ROI), we perform the Gaussian filter for smoothing, and binarization based on Otsu's method [2]. Next, the labeling process is performed, and the largest area detected as lung area.

\section{B. Enhance abnormal shadows based on double-ring filter}

Most of the abnormal shadows in CT images hascircular shape. In addition, because of the use of low-dose CT, the effect of noise should be considered. From these points of view, we use a double-ring filter [3] for emphasis processing, which can enhance the difference in pixel values between the center and the surrounding area to reduce the effect of noise. The output value of the doublering filter is the average value of the inner pixel value minus the average value of the outer pixel value. In this paper, the $7 \times 7$ pixels of a $9 \times 9$ filter are considered.

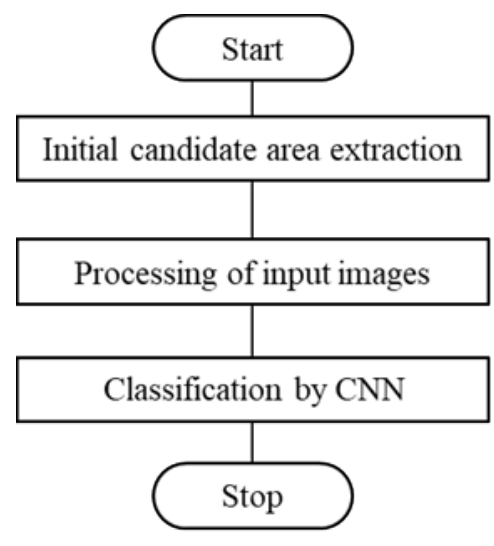

Fig. 1. The flow of CAD system

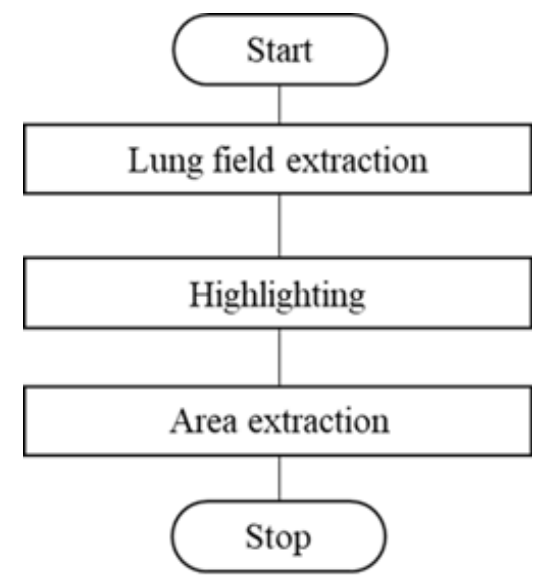

Fig. 2. initial candidate area extraction

\subsection{Input images to $C N N$}

To make it easier to identify the abnormal shadows, we added processing to the images input to the CNN. Specifically, the image before one slice of the candidate region was made into $\mathrm{R}$ components, the image of the candidate region was made into $\mathrm{G}$ components, and the image after one slice of the candidate region was made into B components on the $\mathrm{CNN}$ model. This allows us to obtain a two-dimensional image with spatial information. Figure 4 shows an example of the input image. 


\section{3. $C N N$}

In this paper, we perform transfer learning based on the VGG-16 [4] network structure learned using ImageNet. In addition, we frozen weights up to convolutional layer 10 and retrained after that layers with the generated images. Also, we add a batch normalization [5] behind all convolutional layers to suppress the influence of bias in the data. To adjust the weights of the training, and to suppress over-learning and to obtain global features, we change all the coupled layers to global average pooling [6] and reconstruct the model as shown in Fig.5.

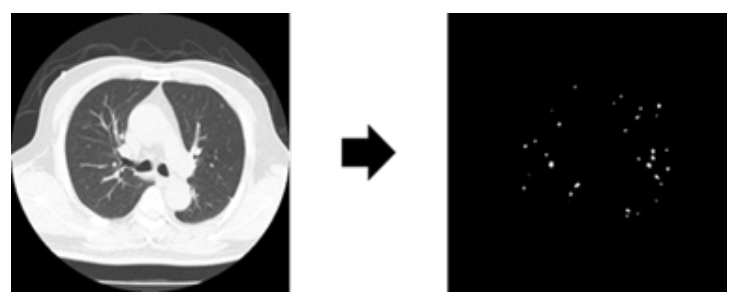

Fig.3 Extraction of candidate areas

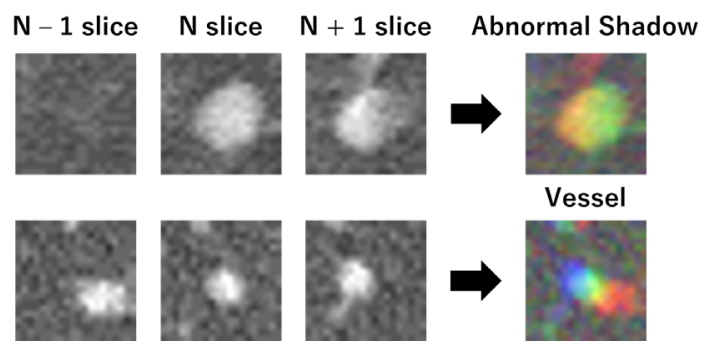

Fig.4 Input images to the $\mathrm{CNN}$

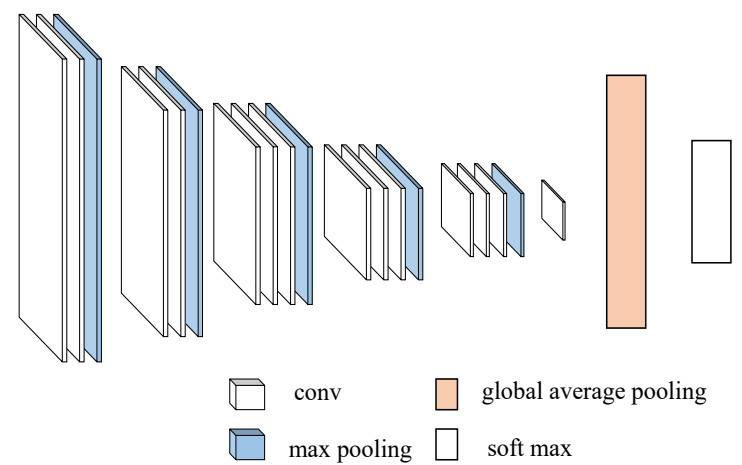

ial Life and Robotics (ICAROB2021), January 21 to 24, 2021

\section{Results}

\subsection{Detail of Dataset}

In this paper, we prepare 13 classes of data sets. One class consists of test and training data. The test data contains 1 abnormal shade and 6 normal tissues. The training data contains 156 abnormal shades and 936 of normal tissue. The training data contains augmented data. Data augment includes vertical and horizontal inversion, mirror image inversion, contrast adjustment and gamma correction.

\subsection{Evaluation metrics}

The performance is evaluated using leave-on-out crossvalidation. Classification accuracy is evaluated by the following equations: TPR(True Positive Rate) \%, and FPR(False Positive Rate) \%, respectively. Here a,b,c,d are shown in Table.1.

$$
\begin{aligned}
& T P R=\frac{a}{a+b} \\
& F P R=\frac{c}{c+d}
\end{aligned}
$$

\subsection{Results}

In this paper, we use the CNN model described in 2.3. We discuss the case where the inter-slice information is reflected in the input image (proposed method) and the case where it is not reflected in the input image as described in 2.2. Table 2 shows classification results. In Table 2, the case where the inter-slice information is reflected in the input image is shown as the proposed method, and the case where it is not reflected is shown as denoted as baseline. As a comparison, we also performed with a method of classifying the SVM [7] with features extracted using $\mathrm{CNN}$, which is effective when the number of images in the dataset is small. The proposed method achieved a TPR of $84.6 \%$, FPR of $15.4 \%$. In contrast, when the input image does not reflect the inter-slice information, the TPR of $53.8 \%$, FPR $15.4 \%$. The SVM is used as another classifier, the TPR of $53.8 \%$, FPR $1.3 \%$ was obtained.

Fig.5 CNN 


\section{Discussion}

We used a CNN model to discriminate the presence or absence of lung cancer in low-dose CT images. The TPR Table.1 Confusion matrix

\begin{tabular}{|l|l|c|c|}
\hline \multicolumn{2}{|c|}{} & \multicolumn{2}{l|}{ Prediction Class } \\
\cline { 3 - 4 } \multicolumn{2}{c|}{} & positive & negative \\
\hline $\begin{array}{l}\text { Actual } \\
\text { Class }\end{array}$ & positive & $a$ & $b$ \\
\cline { 2 - 4 } & negative & $c$ & $d$ \\
\hline
\end{tabular}

Table.2 Experimental results

\begin{tabular}{|c|c|c|}
\hline & TPR\% & FPR $\%$ \\
\hline Proposed method & 84.6 & 15.4 \\
\hline Baseline & 53.8 & 15.4 \\
\hline SVM & 53.8 & 1.3 \\
\hline
\end{tabular}

of the proposed method was $30.8 \%$ higher than that of the baseline. This suggests that reflecting inter-slice information in the input image may be useful. In some cases, abnormal shading could not be detected. The reason may be that what could not be detected was very faint. This can be solved by using an image with enhanced shading in the input image. In addition, we would like to improve the structure of $\mathrm{CNN}$ in the future.

\section{Conclusion}

We developed a CNN-based method for detecting abnormal shadows from low-dose CT images. We achieved a TPR of $84.6 \%$ and FPR of $15.4 \%$. In future, we need to develop a shading enhancement method for the input images and improve the structure of the CNN to further improve the classification accuracy.

\section{References}

1. World Health Organization, Cancer, https://www.who.int/en/news-room/factsheets/detail/Cancer

2. Otsu, "A threshold selection method from gray-level histograms", IEEE Transactions on Systems, Man and Cybernetics, Vol.9, No. 1, pp.62-66, 1979.

3. Y. Hatanaka, et al., "Automated detection algorithm for arteriolar narrowing on fundus images," Proceedings of the 27th Annual International Conference of the IEEE Engineering in Medicine and Biology Society, pp. 286289, 2006.
4. K. Simonyan, "Very deep convolutional networks for large-scale image recognition", arXiv preprint arXiv: 1409. 1556, 2014.

5. Sergey Ioffe Christian Szegedy, "Batch Normalization: Accelerating Deep Network Training by Reducing Internal Covariate Shift”, arXiv:1502.03167, 2015.

6. Min Lin et al., Network In Network" , Cornell University Library, arXiv: 1312.4400, 2014.

7. B. E. Boser et al., "A training algorithm for optimal margin classifiers", Proc. 5th ACM Workshop on Computational Learning Theory, pp.144-152, 1992. 